\title{
The Role of Rumination and Reduced Concreteness in the Maintenance of Posttraumatic Stress Disorder and Depression Following Trauma
}

\author{
Thomas Ehring $\cdot$ Silke Frank $\cdot$ Anke Ehlers
}

Published online: 28 March 2007

(C) Springer Science+Business Media, LLC 2007

\begin{abstract}
Rumination has been linked to posttraumatic stress disorder (PTSD) and depression following trauma. A cross-sectional $(N=101)$ and a prospective longitudinal study $(N=147)$ of road traffic accident survivors assessed rumination, PTSD and depression with self-report measures and structured interviews. We tested the hypotheses that (1) rumination predicts the maintenance of PTSD and depression and (2) reduced concreteness of ruminative thinking may be a maintaining factor. Rumination significantly predicted PTSD and depression at 6 months over and above what could be predicted from initial symptom levels. In contrast to the second hypothesis, reduced concreteness in an iterative rumination task was not significantly correlated with selfreported rumination frequency, and did not consistently correlate with symptom severity measures. However, multiple regression analyses showed that the combination of reduced concreteness and self-reported frequency of rumination predicted subsequent PTSD better than rumination frequency alone. The results support the view that rumination is an important maintaining factor of trauma-related emotional disorders.
\end{abstract}

Keywords: PTSD $\cdot$ Depression $\cdot$ Rumination $\cdot$ Trauma $\cdot$

Concreteness

\section{Introduction}

Posttraumatic stress symptoms are common in the immediate aftermath of traumatic experiences but only a minority of trauma survivors develops chronic psychological problems. It therefore appears important to investigate which variables maintain

T. Ehring $\cdot$ S. Frank $\cdot$ A. Ehlers $(\bowtie)$

Department of Psychology, Institute of Psychiatry, King's College London, P077,

De Crespigny Park, London SE5 8AF, UK

e-mail: a.ehlers@iop.kcl.ac.uk

Present Address:

T. Ehring

Department of Psychology, University of Bielefeld, Bielefeld, Germany

Springer 
posttraumatic stress symptoms (Ehlers \& Steil, 1995; Schnurr, Lunney, \& Sengupta, 2004). Ehlers and Clark (2000) suggested that one important maintaining factor in posttraumatic stress disorder (PTSD) is rumination about the trauma and/or its consequences. ${ }^{1}$ Whereas much of the earlier literature on PTSD subsumed rumination under the re-experiencing symptoms of PTSD (e.g., Greenberg, 1995; Holman \& Silver, 1998), several theorists have recently argued that remembering the trauma (re-experiencing) and repetitively thinking about it (rumination) are phenomenologically and functionally different forms of intrusive cognitions in PTSD (Ehlers \& Clark, 2000; Joseph, Williams, \& Yule, 1997). Intrusive re-experiencing is thought to be due to characteristics of cognitive processing during the trauma and the resulting trauma memory (Brewin \& Holmes, 2003; Ehlers, Hackmann, \& Michael, 2004), whereas rumination is thought to be driven by problematic appraisals and can be regarded as an important cognitive strategy used by individuals with PTSD to control perceived threat (Ehlers \& Clark, 2000; Joseph et al., 1997).

Some preliminary phenomenological studies indeed supported a distinction between rumination and intrusive re-experiencing. Differences were found on a number of dimensions, including content (evaluative thoughts vs. memories), type of cognition (verbal thoughts vs. sensory impressions) and duration (minutes/hours vs. seconds) (Evans, Ehlers, Mezey, \& Clark, in press; Hackmann, Ehlers, Speckens, \& Clark, 2004; Michael, Ehlers, Halligan, \& Clark, 2005; Speckens, Ehlers, Hackmann, Ruths, \& Clark, submitted).

In line with the hypothesis that rumination maintains PTSD, there is preliminary evidence that repetitive and perseverative thinking about issues surrounding the trauma such as its causes, consequences and implications predicts PTSD symptom severity following traumatic events (Clohessy \& Ehlers, 1999; Ehlers, Mayou, \& Bryant, 1998; Michael, Halligan, Clark, \& Ehlers, in press; Murray, Ehlers, \& Mayou, 2002; NolenHoeksema \& Morrow, 1991; Steil \& Ehlers, 2000). Prospective longitudinal studies further found that rumination explains PTSD symptom severity over and above other known predictors (Ehlers et al., 1998), and over and above diagnostic status at initial assessment (Michael et al., in press).

Although past trauma research has largely focused on PTSD, traumatic experiences can also trigger other disorders, especially major depression (e.g., Mayou, Bryant, \& Ehlers, 2001; O'Donnell, Creamer, Pattison, \& Atkin, 2004). There is extensive evidence that rumination is involved in the development and maintenance of depression and is associated with a range of detrimental cognitive, emotional and social effects in dysphoric individuals (for a review see Lyubomirsky \& Tkach, 2004). However, only one published study to date has investigated the role of rumination in the development of depressive symptoms following trauma. Nolen-Hoeksema and Morrow (1991) assessed symptoms of depression in college students who had experienced the Loma Prieta earthquake. The students had filled in a trait measure of depressive rumination 2 weeks prior to the earthquake. The authors found that trait levels of a ruminative

\footnotetext{
${ }^{1}$ Rumination as defined by Ehlers and Clark (2000) includes thoughts about the past as well as the future. It could therefore be argued that these are two different forms of perseverative thinking, namely 'worry' (i.e., recurrent anxious thoughts about the future; see Borkovec, 1994) in addition to 'rumination' (i.e., recurrent negative thoughts about the past and the present; see Papageorgiou \& Wells, 2004). However, for reasons of simplicity and in line with Ehlers and Clark, only the term 'rumination' will be used in this paper to describe perseverative thinking about the trauma and its consequences, including thoughts about the past or present as well as anxious thoughts about the future.
} 
response style to sad mood assessed before the event significantly predicted post-event levels of depression, even when initial symptoms of depression were controlled for.

In the studies described above, PTSD and depressive symptoms were assessed by self-report measures so that it cannot be ruled out that response styles were partly responsible for the observed associations. The present studies tested whether the relationship between rumination and PTSD and depression after trauma can be replicated using structured diagnostic interviews.

Furthermore, the present studies were designed to explore a possible mechanism by which rumination may maintain PTSD. Theories regarding the role of recurrent negative thinking in emotional disorders need to explain two features. First, although recurrent negative thinking has been found to be associated with emotional disorders, repeatedly thinking about one's problems or anticipating possible future threats can also be helpful in reaching one's goals and successfully solving problems (King \& Pennebaker, 1996; Martin \& Tesser, 1996). It therefore appears important to specify how dysfunctional forms of recurrent thinking can be distinguished from functional ones (see also Harvey, Watkins, Mansell, \& Shafran, 2004; Watkins, 2004). Second, rumination and worry are typically described as involuntary and difficult to control. Once ruminative or worrisome thoughts are triggered in individuals with emotional disorders, they tend to persevere for minutes or even hours (Borkovec, 1994; Speckens et al., submitted). It therefore appears necessary to understand the self-perpetuating properties of rumination in individuals with emotional disorders.

A number of different hypotheses have been developed to account for one or both of these features of recurrent negative thinking (e.g., Borkovec, Alcaine, \& Behar, 2004; Papageorgiou \& Wells, 2003; Startup \& Davey, 2001; Wells, 1995). Several authors have argued that functional and dysfunctional forms of recurrent thinking can be distinguished on the basis of the mode in which information is processed. For example, Teasdale (1999) and Watkins (2004) suggested that the detrimental effects of ruminative self-focus are due to a conceptual-evaluative-as opposed to an experiential-mode of processing meaning. Similarly, Borkovec and colleagues described worry as a form of cognitive avoidance that is mainly based on verbal thinking and leads to the suppression of visual imagery as well as the attenuation of physiological and emotional responding to aversive stimuli, thereby inhibiting emotional processing (Borkovec, 1994; Borkovec et al., 2004). Stöber further elaborated this idea in the reduced concreteness hypothesis of worry (Stöber, 1998; Stöber \& Borkovec, 2002). He proposed that worrying leads to reduced imagery because it mainly consists of abstract thoughts that evoke less imagery and physiological and emotional responses than thoughts of more concrete content (see also Paivio, 1986). Although reduced concreteness might help to avoid aversive imagery or strong emotions in the short term, it is thought to maintain recurrent thinking because it is less likely than concrete thinking to produce a specific conclusion and a suitable problem solution, and because it inhibits emotional processing. The latter suggestion is in line with Foa and Kozak (1986) who suggested that emotional processing requires the activation of the relevant emotional memory in order to enable the integration of new information. Thinking about a problem or feared situation in an abstract way may lead to an incomplete activation of the relevant emotional memory, and may thus interfere with emotional processing.

Evidence for the reduced concreteness hypothesis of worry comes from three studies. In two studies, participants were asked to describe problems or worries and their potential consequences, and their answers were rated for levels of concreteness. Stöber, Tepperwien, and Staak (2002, Study 1) found that students' elaborations of 'high-worry' 
topics were less concrete than those of topics that they did not worry much about. In a second study, patients with generalized anxiety disorder provided less concrete worry descriptions than controls (Stöber \& Borkovec, 2002). With successful cognitive behavior therapy, the patients' descriptions became more concrete.

In the third study, Stöber et al. (2002, Study 2) used the 'Catastrophizing Interview' originally developed by Vasey and Borkovec (1992). This interview assesses perseverative thinking about a worrisome topic in an iterative procedure by asking participants to elaborate their concerns repeatedly. The degree of perseverative thinking is operationalized as the number of steps completed in the interview. In addition, worry content can be assessed from the answers given in the interview. The interview has successfully been used by different researchers to test hypotheses regarding the process and content of worry (Davey, Jubb, \& Cameron, 1996; Davey \& Levy, 1998, 1999; Provencher, Freeston, Dugas, \& Ladouceur, 2000; Startup \& Davey, 2001, 2003), depressive rumination (Watkins \& Mason, 2002) and perseverative thinking in insomnia (Harvey \& Greenall, 2003). Stöber et al., (2002) compared the concreteness of answers in the Catastrophizing Interview for topics that the student participants worried about to those that they did not worry about, and found lower concreteness for 'high-worry' topics.

These findings provide preliminary support for the hypothesis that worry is characterized by reduced concreteness. It is conceivable that trauma-related rumination in PTSD may similarly be characterized by reduced concreteness. If this is the case, low concreteness may be one mechanism by which rumination maintains PTSD. According to Ehlers and Clark (2000), recovery from posttraumatic stress symptoms depends on an elaboration of the trauma memory and a modification of problematic appraisals. Both processes require the processing of information on very concrete levels, and thinking about the trauma in an abstract way is unlikely to produce changes in the trauma memory or appraisals.

The studies reported in this paper had two aims. First, we aimed to replicate earlier reports on the role of rumination in predicting PTSD and depression, using both selfreports of symptom severity and structured diagnostic interviews. It was expected that rumination is concurrently and prospectively related to the severity of PTSD and depressive symptoms and that it significantly predicts symptoms at follow-up over and above initial symptom levels. Second, we aimed to test whether the reduced concreteness hypothesis of perseverative thinking applies to rumination in trauma survivors. It was expected that (a) self-reported rumination is significantly related to reduced concreteness of answers in an iterative Rumination Interview that is based on the Catastrophizing Interview, (b) reduced concreteness is significantly related to the perseveration of negative thinking in the interview (i.e., the number of steps) and (c) reduced concreteness is significantly related to symptom levels of PTSD and depression.

\section{Method}

Participants

Participants had attended King's College Hospital's Accident and Emergency (A\&E) Department, London, following injury in a road traffic accident. Inclusion criteria were: injury in a road traffic accident as a driver, passenger, motorcyclist, or cyclist; injuries 
exceeded triage category 'blue' (very mild injuries); age between 18 and 65; address in greater London. Exclusion criteria were: left before receiving medical treatment; attended the A\&E Department more than 3 days after the accident; current psychosis or suicidality.

\section{Study 1: cross-sectional investigation}

The sample comprised 101 participants $(56 \%$ male; age: $M=34.95, \mathrm{SD}=10.60)$ who had experienced a road traffic accident between 3 and 12 months prior to their participation in the study. These participants were interviewed on one occasion. Twenty-three percent of participants had experienced the accident as a car driver, $22 \%$ as a passenger, $29 \%$ as a motorcyclist and $27 \%$ as a bicyclist. The A\&E triage scores were used to approximate injury severity in this sample. Sixty-five percent of participants received the triage category 'green' (minor injuries), 29\% the category 'yellow' (urgent) and 6\% the category 'red' (life-threatening). The majority of participants (85\%) were discharged from hospital within $24 \mathrm{~h}$ of their accident. Detailed information about sample and accident characteristics is given elsewhere (Ehring, Ehlers, \& Glucksman, in press).

\section{Study 2: prospective longitudinal investigation}

The second sample comprised 147 participants (67\% male; age: $M=35.17, \mathrm{SD}=9.40$ ). Participants were either approached by a researcher on the day of their accident while attending the A\&E Department $(n=53)$ or contacted via letter and telephone within the first week following their accident $(n=94)$. Results for these subgroups did not differ. Participants attended an interview session at 2 weeks after the accident, and were followed for 6 months. Thirty percent of participants in Study 2 had experienced the accident as car drivers, $16 \%$ as passengers, $38 \%$ as motorcyclists and $26 \%$ as bicyclists. Sixty percent of participants received the triage category 'green' (minor injuries), 36\% the category 'yellow' (urgent) and 4\% the category 'red' (life-threatening). The mean injury severity score (ISS) (Baker, O'Neil, Haddon, \& Long, 1974) in this sample was $2.12(\mathrm{SD}=2.34, \min =0, \max =13)$. As in Study 1 , the majority of participants $(89 \%)$ were discharged from hospital within $24 \mathrm{~h}$ of the accident. Detailed information about sample and accident characteristics is given elsewhere (Ehring, Ehlers, \& Glucksman, in preparation).

Table 1 shows the prevalences of acute stress disorder (ASD), PTSD and major depression in the two samples, as assessed with the Structured Clinical Interview for the DSM-IV (SCID; First, Spitzer, Gibbon, \& Williams, 1996). In both studies, a high comorbidity between the disorders was found: Forty-one percent (Study 1) and 52\% (Study 2) of participants with ASD/PTSD at the initial assessments also met criteria for major depression; and 64\% (Study 1) and 86\% (Study 2) of participants with major depression also suffered from PTSD.

Measures and tasks

Diagnoses and symptom severities

In both studies, PTSD and major depression were assessed with the SCID (First et al., 1996). In Study 2 only, the Acute Stress Disorder Scale (ASDS; Bryant \& Harvey, 2000) 
Table 1 Prevalences of PTSD and major depression in the two studies according to the structured clinical interview for DSM-IV

\begin{tabular}{|c|c|c|c|}
\hline \multicolumn{2}{|l|}{ Study $1(N=101)$} & \multicolumn{2}{|l|}{ Study 2} \\
\hline \multirow[t]{2}{*}{ Diagnosis } & Prevalence $(n, \%)$ & Diagnosis & Prevalence $(n, \%)$ \\
\hline & & \multicolumn{2}{|c|}{ Assessment at 2 weeks $(N=147)$} \\
\hline PTSD & $22(21.8 \%)$ & ASD & $23(15.5 \%)$ \\
\hline \multirow[t]{5}{*}{ Major depression } & $11(10.9 \%)$ & PTSD $^{\mathrm{a}}$ & $33(22.4 \%)$ \\
\hline & & Major depression & $14(9.6 \%)$ \\
\hline & & \multicolumn{2}{|c|}{ Assessment at 6 months $(N=140)$} \\
\hline & & PTSD & $17(12.1 \%)$ \\
\hline & & Major depression & $11(7.8 \%)$ \\
\hline
\end{tabular}

PTSD posttraumatic stress disorder, $A S D$ acute stress disorder

${ }^{a}$ Without criterion $\mathrm{E}$ (duration for at least 1 month)

was conducted as an interview at the 2-week assessment. On the basis of the participant's responses to the ASDS, the researcher rated the presence or absence of DSM-IV symptom criteria for ASD. Interrater-reliabilities for the SCID and ASDS interviews were high (PTSD: $\kappa=.82$; ASD: $\kappa=.93$; major depression: $\kappa=1, N=56$ randomly chosen interviews from this and a related study, two raters). The SCID manual states that $\kappa^{\varsigma}$ s above .70 indicate good reliability (First et al., 1996).

Posttraumatic stress disorder symptom severity was assessed using the Posttraumatic Diagnostic Scale (PDS; Foa, Cashman, Jaycox, \& Perry, 1997), a validated and widely used self-report measure of PTSD symptom severity. The Beck Depression Inventory (BDI; Beck, Rush, Shaw, \& Emery, 1979), a standardized questionnaire of established reliability and validity, assessed the severity of depressive symptoms.

\section{Self-report rumination measures}

The rumination subscale of the Responses to Intrusions Questionnaire (RIQ) was used to assess rumination about the trauma and/or its consequences. This self-report questionnaire assesses different aspects of trauma survivors' responses to intrusive memories. It was developed in a series of studies (Clohessy \& Ehlers, 1999; Halligan, Clark, \& Ehlers, 2002; Murray et al., 2002; Steil \& Ehlers, 2000) and has shown good reliability and predictive validity. The rumination subscale consists of eight items (e.g., I think about why the event happened to me; I think about how life would have been different if the event had not occurred; Study 1: $\alpha=.80$; Study 2: $\alpha=.86$ ), rated on a scale from 0 (never) to 3 (always).

The rumination scale of the Response Style Questionnaire (RSQ; Nolen-Hoeksema \& Morrow, 1991) was used to assess depressive rumination defined as perseverative thinking about one's symptoms of depression and possible causes and consequences of these symptoms (Nolen-Hoeksema, 2004). The full rumination scale of the RSQ has been used widely in clinical as well as non-clinical populations and has demonstrated high reliability and validity (Luminet, 2004; Nolen-Hoeksema, 2004). A ten-item short version of the questionnaire was found to show psychometric properties equivalent to the full scale (S. Nolen-Hoeksema, personal communication) and was therefore used in this project. Participants rated each item (e.g., I think about how sad I feel) on a scale from 1 (never) to 4 (always), reflecting how often they had this thought when they were feeling sad, down or depressed. The internal consistency of the scale was $\alpha=.92$ in Study 1 and $\alpha=.89$ in Study 2 . 


\section{Rumination Interview}

The Rumination Interview generated the material for ratings of the concreteness of the participants' ruminative thoughts. It was adapted from the Catastrophizing Interviews used by Vasey and Borkovec (1992) and Davey and Levy (1998). To familiarize participants with the procedure, they were first taken through an example of the interview format, using a concern unrelated to the trauma (concern about being late for work). Participants were then instructed to continue answering the interview questions until they had sufficiently explored their concern. This instruction was chosen as earlier research investigating the effects of varying stop rules on perseveration in the task have found this condition to best distinguish between groups of high vs. low worriers or ruminators ('as many as can' stop rule; Startup \& Davey, 2001, 2003; Watkins \& Mason, 2002).

In the first part of the interview (trauma-related rumination), participants were asked to identify their main current concern related to the accident ('What is it that concerns you most about the accident?'). When the participant had named their concern ' $X$ ', the interview started with the question 'What is it that concerns you most about X?'. The answer Y was followed by the interviewer's question 'What is it that concerns you most about Y?' and so forth. After 7,14 and 21 answers ('steps'), the experimenter reminded participants that they could end the interview when they had reached the goal of sufficiently exploring their concern. The interview continued until participants indicated that they wanted to end the procedure.

For the second part of the interview (depressive rumination), participants were asked to identify a topic of current or recent sad mood. The interview followed the same procedure, with the question asked being 'Why is it that X makes you feel sad?'. This part was identical to the interview used by Watkins and Mason (2002).

Some participants were unable to identify a current concern related to the trauma or a topic of current or recent sad mood. The number of participants for whom Rumination Interview data was available was $n=83$ for the trauma-related rumination part and $n=84$ for the depressive rumination part in Study 1; and $n=114$ for the trauma-related rumination part and $n=96$ for the depressive rumination part in Study 2. Participants with vs. those without disorders were equally likely to be unable to identify a topic for the interview (all $\chi^{2}<.93$, all $P>.41$ ).

The interviews were audio taped and transcribed verbatim. The number of steps generated by each participant in each of the interview parts was calculated. A graduate student of psychology rated the concreteness of the participants' answers from the interview transcripts on the scale developed by Stöber and colleagues (Stöber \& Borkovec, 2002; Stöber et al., 2002), which consists of five categories: 1 (abstract), 2 (somewhat abstract), 3 (neither-nor), 4 (somewhat concrete) and 5 (concrete). The category 'abstract' is defined as 'indistinct, cross-situational, equivocal, unclear, aggregated'. The category 'concrete' is defined as 'distinct, situationally specific, unequivocal, clear, singular'. The rater was blind to the participants' diagnostic status and did not know which interview parts were generated by the same individual. Each answer was rated separately, and mean concreteness scores for each of the two interview parts (trauma-related rumination and depressive rumination) were computed.

In order to assess the inter-rater reliability of the concreteness ratings, 20 randomly chosen trauma-related Rumination Interviews and 20 randomly chosen depressive Rumination Interviews were independently rated by the first author. The single measure 
intraclass correlation coefficient for all interviews was .79 (.86 for the trauma-related rumination part and .71 for the depressive rumination part). This is similar to the reliability of ratings reported by Stöber and Borkovec (2002).

Two different Rumination Interview parts were used to increase the reliability of assessment and to investigate possible content specificity of rumination about the trauma and depressive rumination. However, largely identical results were found for the two parts. Therefore, the two interview parts were combined and the mean number of steps as well as mean concreteness ratings computed. ${ }^{2}$ For reasons of brevity, only results for these overall scores will be reported.

Design and procedure

The study was approved by the local research ethics committees and participants gave written informed consent.

\section{Study 1}

After agreeing to take part in the study, participants received a pack of questionnaires, including the rumination and the symptom severity measures as well as some measures unrelated to the analyses presented here. Participants filled in the questionnaires on the day before attending an assessment session. During the session, the participants completed the Rumination Interview and the SCID, as well as some additional tasks unrelated to the analyses presented here. All assessments were conducted by the first author. Participants received $£ 30$ as a reimbursement for their time.

\section{Study 2}

The study comprised four assessments. At approximately 2 weeks posttrauma, participants attended a session, which was identical to the session in Study 1. Symptom severity measures were repeated at three postal follow-up assessments $(1,3$ and 6 months following the accident). At the 6-month follow-up, the SCID was also repeated by telephone. Self-report rumination measures were repeated at 1 month posttrauma because a previous study (Murray et al., 2002) had suggested that the predictive power of rumination increases with time. The session as well as the first follow-up assessment also contained some additional tasks and/or questionnaires unrelated to the analyses presented here. Participants received $£ 50$ as a reimbursement for their time.

Data analyses

Variables were transformed to normal via square root or logarithmic transformations prior to analyses if necessary. Diagnostic groups were compared with $t$-tests. Pearson's product-moment correlation coefficients were computed to investigate the relationship between variables of interest.

\footnotetext{
${ }^{2}$ For nine participants, only data from one of the interview parts was available and therefore the available score was used in the analyses. Excluding these participants from the analyses did not change the results.
} 


\section{Results}

Self-report rumination measures

\section{Relationship between self-reported rumination and outcome measures}

Table 2 shows the group differences in rumination between participants with and without disorders following the trauma. The $t$-tests showed that participants with ASD, PTSD or major depression reported more rumination about the trauma (RIQ) and more depressive rumination (RSQ) than participants without these disorders. Table 3 shows the correlations between self-reported rumination and symptom severity measures at the different time points across both studies. Rumination about the trauma (RIQ) as well as depressive rumination (RSQ) correlated significantly with PTSD and depressive symptom severities at all assessments.

For Study 2, partial correlations tested whether rumination predicts symptoms at 6 months over and above what can be predicted from initial symptom levels. Traumarelated rumination measured at 2 weeks predicted PTSD symptom severity at 6 months over and above symptom levels at 2 weeks (partial $r=.27, P<.01$ ). Similarly, trauma-related rumination measured at 1 month predicted PTSD symptom severity at 6 months over and above symptom levels at 1 month (partial $r=.38$, $P<.001)$. Likewise, self-reported depressive rumination measured at 2 weeks predicted BDI scores at 6 months over and above what was predicted from initial depressive symptoms at 2 weeks (partial $r=.30, P<.01$ ), and similarly for the 1 -month measures (partial $r=.27, P<.01$ ).

\section{Additional analyses}

Additional analyses were conducted to rule out the possibility that correlations between the rumination scales and the PDS were due to the participants' failure to distinguish between re-experiencing and rumination. We computed adjusted PTSD symptom severity scores, omitting the re-experiencing items. All correlations and partial correlations between the rumination scales and PTSD severity remained significant for the adjusted score and were almost identical to those shown in Table 3 .

We further computed partial correlations in order to test whether there were specific associations between trauma-related rumination and PTSD on the one hand, and between depressive rumination and depression on the other hand. For concurrent assessments, PTSD symptom severities were still significantly correlated with both trauma-related $(r$ 's $>.37)$ as well as depressive rumination scores $(r$ 's $>.21)$ in both samples when statistically controlling for symptom levels of depression. Similarly, BDI scores still showed significant correlations with depressive rumination $(r$ 's $>.50)$ and trauma-related rumination $(r$ 's $>.36)$ when controlling for PDS scores. However, for the prediction of future symptom levels in Sample 2, specific associations emerged. PTSD symptom severities at 6 months follow-up were only significantly predicted by trauma-related rumination (partial $r=.35, P<.001$ ), but not by depressive rumination at initial assessment (partial $r=.05, P=.60$ ) when symptom levels of depression were partialled out. Similarly, only depressive rumination (partial $r=.26, P<.01$ ), but not trauma-related rumination (partial $r=.09, P=.32$ ), predicted BDI scores at 6 months when PDS scores were partialled out. 


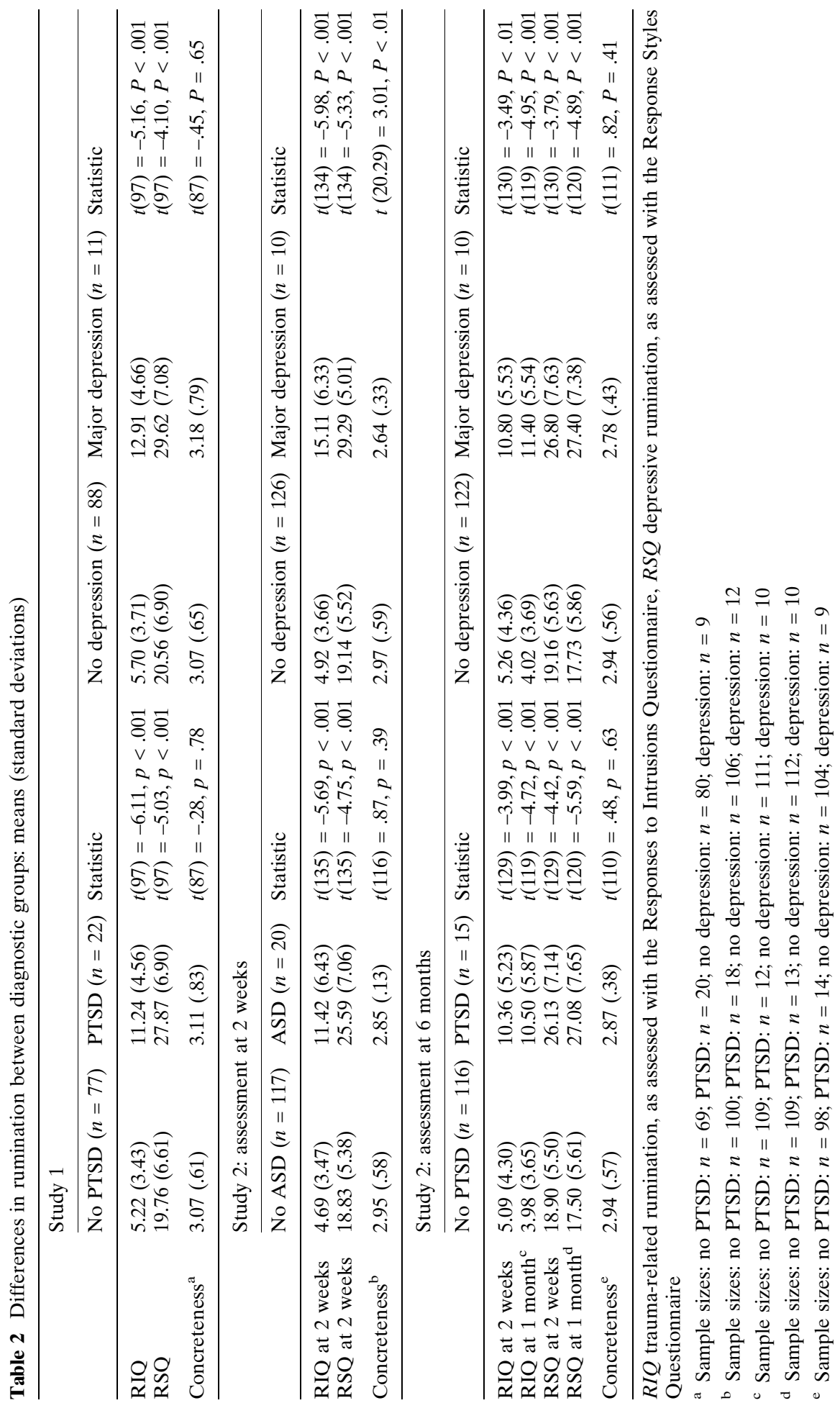




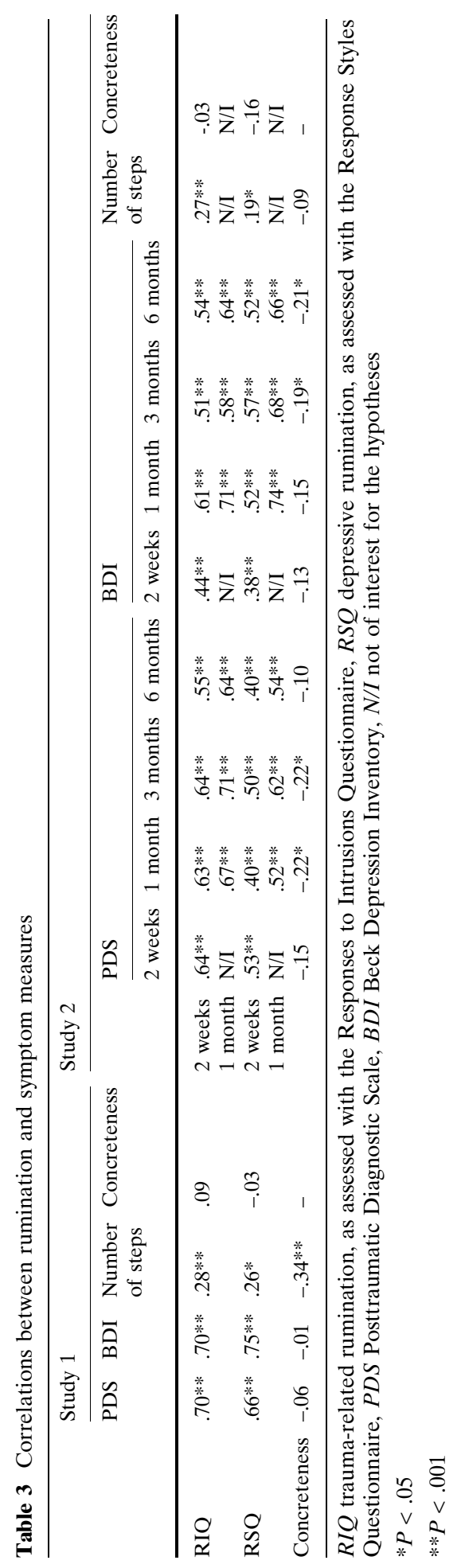


Relationship between rumination measures and stability of rumination scores

Trauma-related (RIQ) and depressive rumination (RSQ) scores correlated substantially at all assessments, Study 1: $r=.57, P<.001$; Study 2, 2 weeks: $r=.55, P<.001$, 1 month: $r=.69, P<.001$.

In order to test the stability of rumination scores in Study 2, analyses of variance with 'diagnostic group' as between-subject factor and 'time of assessment' as within-subject factor were conducted. For trauma-related rumination scores, a significant interaction between 'ASD at 2 weeks' and 'time of assessment' was found, $F(1,119)=5.44$, $P<.05$. Follow-up tests showed that the degree of trauma-related rumination (RIQ) significantly decreased from 2 weeks to 1 month in participants without ASD, $M=4.55$, $\mathrm{SD}=3.45$ vs. $M=3.71, \mathrm{SD}=3.38 ; t(105)=4.29, P<.001$, whereas participants with ASD did not show a change in rumination scores during this interval, $M=10.40$, $\mathrm{SD}=5.58$ vs. $M=10.93, \mathrm{SD}=5.43, t(14)=-.75, P=.47$. For depressive rumination scores (RSQ), there was no significant main effect of 'time of assessment', $F(1,121)=.55, P=.46$ nor a significant interaction between 'depression at 2 weeks' and 'time of assessment', $F(1,121)=.23, P=.63$.

\section{Rumination Interview}

\section{Number of steps}

In Study 1, participants generated a mean of $M=7.39$ steps in the trauma-related rumination part, $\mathrm{SD}=3.65, \min =2, \max =20$, and $M=6.33$ steps in the depressive rumination part of the Rumination Interview, $\mathrm{SD}=3.69, \min =2, \max =23$. For Study 2 , the numbers were a mean of $M=7.11$ steps in the trauma-related rumination part, $\mathrm{SD}=4.13, \min =2, \max =24$, and $M=6.71$ steps in the depressive rumination part, $\mathrm{SD}=3.85, \min =2, \max =25$. As shown in Table 3 , the mean number of steps in the interviews correlated significantly with self-report levels of rumination.

\section{Concreteness ratings}

In neither study did concreteness of answers in the Rumination Interview correlate with self-reported rumination. In Study 1, but not in Study 2, mean concreteness ratings were significantly correlated with the number of steps in the interview.

Study 1 found neither significant correlations between mean concreteness ratings and symptom severity scores (see Table 3), nor significant group differences (see Table 2).

In Study 2, concreteness in the Rumination Interview at 2 weeks was significantly negatively related to depressive symptom severity at 3 and 6 months (Table 3 ). Similarly, there were significant differences between participants with and without major depression in concreteness levels at 2 weeks (Table 2). For PTSD symptoms, there were no significant correlations (Table 3) or group differences (Table 2) at 2 weeks or 6 months. Concreteness at 2 weeks, however, was negatively related to PTSD symptom severity at 1 and 3 months.

Regression analyses

In order to test whether concreteness of ruminative thinking improves the prediction of symptom levels of PTSD or depression in comparison to the prediction by the frequency 
Table 4 Regression analyses: predicting concurrent and subsequent PTSD severity by self-reported rumination and concreteness at 2 weeks

\begin{tabular}{|c|c|c|c|c|c|c|c|c|c|c|}
\hline & \multirow{2}{*}{\multicolumn{2}{|c|}{$\frac{\text { Study } 1}{\text { PDS scores }}$}} & \multicolumn{8}{|c|}{ Study 2} \\
\hline & & & \multicolumn{2}{|c|}{$\begin{array}{l}\text { PDS at } \\
2 \text { weeks }\end{array}$} & \multicolumn{2}{|c|}{$\begin{array}{l}\text { PDS at } \\
1 \text { month }\end{array}$} & \multicolumn{2}{|c|}{$\begin{array}{l}\text { PDS at } \\
3 \text { months }\end{array}$} & \multicolumn{2}{|c|}{$\begin{array}{l}\text { PDS at } \\
6 \text { months }\end{array}$} \\
\hline & $\beta$ & $\Delta R^{2}$ & $\beta$ & $\Delta R^{2}$ & $\beta$ & $\Delta R^{2}$ & $\beta$ & $\Delta R^{2}$ & $\beta$ & $\Delta R^{2}$ \\
\hline Step 1 & & $.47 * *$ & & $.43^{* *}$ & & $.29 * *$ & & $.29 * *$ & & $.28 * *$ \\
\hline $\begin{array}{l}\text { Trauma-related rumination } \\
\text { (RIQ) }\end{array}$ & $.68^{* *}$ & & $.65^{* *}$ & & $.54 * *$ & & $.54 * *$ & & $.53 * *$ & \\
\hline Step 2 & & $.03^{*}$ & & .01 & & $.03^{*}$ & & $.04 *$ & & .01 \\
\hline $\begin{array}{l}\text { Trauma-related rumination } \\
\text { (RIQ) }\end{array}$ & $.71 * *$ & & $.65 * *$ & & $.53 * *$ & & $.53 * *$ & & $.53 * *$ & \\
\hline Concreteness ratings & $-.18^{*}$ & 01 & .11 & 00 & $-.17 *$ & 00 & $-.19 *$ & 00 & -.12 & $03 *$ \\
\hline Trauma-related rumination & $.76^{* *}$ & & $.65^{* *}$ & .00 & $.53 * *$ & .00 & $.53 * *$ & .00 & $.53 * *$ & .05 \\
\hline Concreteness ratings & $-.17 *$ & & -.13 & & $-.18^{*}$ & & $-.20 *$ & & -.15 & \\
\hline Interaction & -.13 & & .04 & & -.02 & & -.03 & & $-.17 *$ & \\
\hline
\end{tabular}

$P D S$ Posttraumatic Diagnostic Scale, $R I Q$ trauma-related rumination, as assessed with the Responses to Intrusions Questionnaire

$* P<.05$

$* * P<.001$

of rumination alone, we conducted hierarchical regression analyses with symptom scores as dependent variables. In the first step, $z$-transformed self-reported rumination scores were entered, followed by $z$-transformed concreteness scores in the second step and the interaction between the two (product of the $z$-transformed scores) in the third step.

In all analyses with PTSD symptom scores as dependent variable, the frequency of trauma-related rumination entered in the first step significantly predicted symptom severity. In all but one analysis the prediction of PTSD symptom severities could significantly be improved by either concreteness scores or the interaction between concreteness scores and self-reported rumination entered as additional predictor variables, although the additional amount of variance was small and ranged between 3 and $4 \%$ (see Table 4).

Regression analyses with symptom levels of depression as dependent variables only showed significant effects for the self-reported frequency of depressive rumination. However, neither concreteness scores nor the interaction between concreteness and self-reported rumination scores significantly improved the prediction of depressive symptom severities (all $|\beta|<.17$, all $p \mathrm{~s}>.06$ ).

\section{Discussion}

The first aim of this study was to replicate earlier findings regarding the role of rumination in the maintenance of PTSD and depression following trauma. In line with previous studies (e.g., Ehlers et al., 1998; Murray et al., 2002), self-reported rumination about the trauma was significantly and substantially correlated with the severity of PTSD symptoms. Rumination was not only related to concurrent PTSD symptoms, but 
also predicted subsequent symptoms at 6 months. Rumination predicted a substantial amount of the variance (between 36 and $50 \%$ ) of PTSD symptom severity. The findings extended those of previous studies in that rumination not only predicted self-reported PTSD symptoms, but also a diagnosis of ASD at 2 weeks, and a diagnosis of PTSD at 6 months after the traumatic event. In addition, rumination predicted the severity of PTSD symptoms over and above what could be predicted from initial symptom levels. In sum, the results of the present studies clearly support the view that rumination is an important maintaining factor of PTSD following trauma, as suggested by recent theoretical accounts (Ehlers \& Clark, 2000; Joseph et al., 1997).

In an earlier study by Murray et al. (2002), rumination assessed at 1-week posttrauma was found to predict PTSD symptom levels at the 6-month follow-up to a much lower degree than rumination assessed at 1 month after the event. In contrast to this earlier finding, the present study did not find differences in the predictive power of rumination assessed at 2 or 4 weeks. Both showed high correlations with PTSD severity at follow-up. This suggests that rumination is a powerful predictor from 2 weeks onwards.

This finding is of considerable interest for the early detection of people at risk for chronic PTSD. Research into early intervention following trauma has shown discouraging results for debriefing interventions that are offered to every trauma survivor, whereas a course of cognitive-behavioral interventions with individuals at high risk of chronic PTSD has been shown to be effective (for a review see McNally, Bryant, \& Ehlers, 2003). The present study suggests that the assessment of rumination might help improve the early identification of trauma survivors in need of early intervention, given that rumination was highly predictive of PTSD at 6 months and that it predicted over and above initial symptom levels. This idea is further supported by the finding that the degree of trauma-related rumination remained high between 2 weeks and 1 month in participants with ASD whereas participants without the disorder showed a reduction in the frequency of ruminative thinking during this interval.

In the past, trauma-related rumination and re-experiencing symptoms have sometimes been treated as a unitary phenomenon (e.g., Greenberg, 1995; Holman \& Silver, 1998). However, recent theoretical models of PTSD (Ehlers \& Clark, 2000; Joseph et al., 1997) as well as results from recent phenomenological studies (Evans et al., in press; Michael et al., 2005, in press; Speckens et al., submitted) suggest that rumination and re-experiencing are conceptually and phenomenologically distinct. This view is supported by our findings that rumination still predicted the maintenance of PTSD when initial symptoms were statistically controlled for and that the size of the correlations did not change when PTSD symptom severity was calculated omitting the re-experiencing symptoms. Thus, the relationship between rumination and PTSD found in this study cannot be explained by a possible lack of discrimination between rumination and re-experiencing in the participants' answers.

Although there is extensive evidence that rumination is involved in the development and maintenance of depression (see Lyubomirsky \& Tkach, 2004 for a review), only one study to date has investigated the role of rumination in the development of depression following trauma (Nolen-Hoeksema \& Morrow, 1991). In this study, depressive rumination assessed prior to the traumatic experience significantly predicted posttrauma symptoms of depression. The present results extend this finding by showing that levels of depressive rumination assessed after the trauma are also significantly associated with concurrent and subsequent levels of depression. In addition, it could be shown that rumination assessed shortly after the trauma predicts depression at follow-up over and 
above initial symptom levels and that rumination not only predicts self-reported levels of depressive symptoms but also a diagnosis of major depression.

Rumination has been identified as a maintaining factor in a number of emotional disorders and it remains unclear whether different types of perseverative thinking identified in different emotional disorders represent similar or different processes (Harvey et al., 2004). The present results suggest a relationship between trauma-related rumination and depressive rumination. The RIQ and RSQ were moderately to highly correlated, and both measures predicted both PTSD and depression. These findings may have been influenced by the high comorbidity between PTSD and depression in the present samples, as nearly all participants with depression also met criteria for PTSD or subthreshold PTSD. The nature of the samples thus did not allow a direct comparison between rumination in trauma survivors who have PTSD, but no depression, and those who have depression, but no PTSD. However, partial correlations in the prospective study provided preliminary evidence for a specific association between trauma-related rumination and PTSD symptom severity on the one hand (controlling for depressive symptoms), and between depressive rumination and severity of depressive symptoms on the other hand (controlling for PTSD symptoms). It would be desirable in future studies to identify groups of trauma survivors that are more clearly separated by their diagnostic status, for example, by including mixed groups of trauma survivors who have suffered loss or threat to life.

Despite evidence that rumination is involved in the maintenance of emotional disorders following trauma, to our knowledge, no published study to date has investigated the process by which rumination might maintain posttraumatic stress symptoms. Self-report measures used to date mainly assess the frequency and content of rumination and therefore appear to be of limited use when studying the processes underlying perseverative thinking. The present studies therefore adapted the Catastrophizing Interview to investigate process characteristics of rumination in trauma survivors. The original paradigm has repeatedly been shown to be useful in studying factors underlying chronic worry (e.g., Davey et al., 1996; Startup \& Davey, 2001). In the studies reported here, the number of steps in the Rumination Interview was significantly related to self-reported rumination, providing initial evidence that perseveration in the interviews is indeed related to naturally occurring perseverative thinking. However, as in most earlier studies (e.g., Startup \& Davey, 2001; Watkins \& Mason, 2002), correlations were only modest.

The answers given in the Rumination Interview were analyzed to test the reduced concreteness theory of recurrent negative thinking in emotional disorders (Stöber, 1998) in the context of trauma-related PTSD and depression. Three different hypotheses were derived from the theory. First, it was expected that recurrent negative thinking is characterized by reduced concreteness. This hypothesis had found initial empirical support in studies of non-clinical worry and generalized anxiety disorder (Stöber \& Borkovec, 2002; Stöber et al., 2002). The present studies failed to show a relationship between self-reported rumination and level of concreteness in the Rumination Interview. Differences between the present results and Stöber et al.'s findings may be due to differences in methodology, or differences between worry about future events and rumination about a past event. As to methodological differences, Stöber et al.'s positive results with the Catastrophic Interview technique were based on within-subject comparisons showing that the (non-clinical) participants' thinking about topics that they worried about was less concrete than their thinking about non-worry topics. The present studies used between-subject and correlational analyses of rumination about the same topic, 
which may have less power than a within-subject comparison. Furthermore, in contrast to Stöber et al.'s study (2002), all participants were interviewed about a recent very upsetting event, and this may have led to a reduced range in concreteness ratings compared to the range of concreteness for worry and non-worry topics in Stöber et al.'s study.

The results may also point to possible differences between worry and rumination. Worry and rumination have so far mainly been distinguished on the basis of their content, in that rumination is typically associated with thinking about the past, whereas worry represents anxious thoughts about future events (Fresco, Frankel, Mennin, Turk, \& Heimberg, 2002; Segerstrom, Tsao, Alden, \& Craske, 2000). The anticipation of future events (as in worry) may lend itself to a greater range of levels of concreteness than thoughts about past experiences that have already happened (as in rumination), which are by definition more concrete than future events. The investigation of concreteness vs. abstractness of thinking about the past might therefore need a more fine-grained analysis than reflected by the concreteness rating scale developed by Stöber and colleagues. This may apply especially when looking at concerns related to a trauma, which is a specific event with sometimes very concrete worrying consequences such as physical injuries and the need for further operations.

A second hypothesis derived from the reduced concreteness theory was that reduced concreteness is involved in the perseveration of negative thinking in individuals suffering from emotional disorders. In line with this idea, Study 1 showed a significant relationship between the number of steps generated in the Rumination Interview and concreteness ratings. However, Study 2 did not replicate this result. It is conceivable that the observed relationship with reduced concreteness only applies to chronic forms of rumination, as the negative findings in Study 2 were obtained only 2 weeks after the event, when the event may have been very prominent on all participants' minds. Thus, the findings of Study 1 need replication before further conclusions can be drawn.

Finally, we hypothesized that if functional and dysfunctional ways of recurrent thinking about problems or negative experiences differ in concreteness, then reduced concreteness should predict the severity of psychological problems after trauma. This hypothesis received some, but overall weak support in Study 2, which found significant group differences in concreteness between participants with and without major depression at 2 weeks after the accident, and correlations between low concreteness at 2 weeks and severity of depressive symptoms at 3 and 6 months. There were also some correlations between low concreteness and PTSD symptom severity. Overall, the correlations were small. The hypothesis received some further support as multiple regression analyses showed that PTSD symptoms at 6 months were better predicted by the combination of the frequency of trauma-related rumination and reduced concreteness of ruminative thinking at initial assessment than by rumination frequency alone. Overall, the results were disappointing and only showed weak support for the view that reduced concreteness of thinking about the trauma is associated with the maintenance of depression and PTSD following trauma. The results need to be interpreted with great caution as correlations and group differences were non-significant for some of the time points and concreteness in the rumination task did not correlate with self-reported rumination. More research is needed before any firm conclusions regarding the role of reduced concreteness in trauma-related rumination can be drawn. Future studies should especially aim to improve the assessment of concreteness. The measure used in this study might have lacked sensitivity as some of the problems that participants worried about were by definition concrete (physical and financial problems), and the measure 
only consisted of one global rating. Thus, a refined assessment of concreteness may show stronger relationships with self-reported rumination and with psychopathology. In addition, alternative hypotheses regarding the processes by which rumination maintains PTSD should be developed and tested.

In sum, results from the present studies replicated and extended earlier findings regarding the role of rumination in the maintenance of emotional disorders following trauma. Rumination at 2 weeks and 1 month after the trauma appears to be an important predictor of PTSD. In addition, there was very preliminary support for a role of the style or mode of ruminative thinking. However, the hypotheses that frequent rumination and perseveration in the Rumination Interviews are associated with reduced concreteness were only partially supported. More research is needed to replicate and extend these results as well as clarify the disparate findings between the two studies.

A number of limitations of the studies are noteworthy. First, although a prospective longitudinal design was used in Study 2, the results remain correlational. Future studies are needed that manipulate rumination and its characteristics such as concreteness experimentally and test the effect of these manipulations on symptom severities. Second, the high comorbidity between PTSD and depression in the samples studied did not allow a direct comparison between rumination in different diagnostic groups. Future studies comparing clearly distinct diagnostic groups are needed to investigate similarities and differences between different types of perseverative thinking in PTSD and depression. Third, other measures of concreteness may have shown stronger relationships with self-reported rumination and symptom measures. Finally, it remains unclear whether low concreteness is an important dimension underlying dysfunctional forms of recurrent negative thinking. Alternative approaches suggest that functional and dysfunctional ways of thinking about problems or negative experiences can rather be distinguished by the amount of imagery vs. verbal processes involved (Borkovec, 1994; Borkovec et al., 2004) or the degree of conceptual-evaluative vs. experiential self-focus (Teasdale, 1999; Watkins, 2004). Although these views appear to be related, future theoretical as well as empirical work is needed to clarify the relationship between these processes and isolate the most relevant dimensions involved in the maintenance of PTSD and depression after trauma. Such research may point toward possible ways of directly targeting dysfunctional rumination in treatment.

Acknowledgments The study was funded by a Wellcome Prize Studentship to T.E. and a Wellcome Principal Research Fellowship to A.E. We are grateful to Inga Böllinghaus, Anke Weidmann, Johanna Hissbach, Stephanie Spengler, Jennifer Baumeister, Ines Sengstock and Soni Mutschelknauss for their help with data collection and interview transcripts. We would like to thank Dr Edward Glucksman and the staff at King's College Hospital A\&E Department for their collaboration.

\section{References}

Baker, S. P., O’Neil, B., Haddon, W. J. E. A., \& Long, W. B. (1974). The injury severity score: A method for describing patients with multiple injuries and evaluating emergency care. Journal of Trauma, 14, 187-196.

Beck, A. T., Rush, A. J., Shaw, B. F., \& Emery, G. (1979). Cognitive therapy for depression. New York, NY: Guilford.

Borkovec, T. D. (1994). The nature, functions, and origins of worry. In G. C. L. Davey \& F. Tallis (Eds.), Worrying: Perspectives on theory, assessment and treatment (pp. 5-33). Chichester, UK: Wiley.

Borkovec, T. D., Alcaine, O. M., \& Behar, E. (2004). Avoidance theory of worry and generalized anxiety disorder. In R. G. Heimberg, C. L. Turk, \& D. S. Mennin (Eds.), Generalized anxiety disorder: Advances in research and practice (pp. 77-108). New York, NY: Guilford.

Springer 
Brewin, C. R., \& Holmes, E. A. (2003). Psychological theories of posttraumatic stress disorder. Clinical Psychology Review, 23, 339-376.

Bryant, R. A., \& Harvey, A. G. (2000). Acute stress disorder: A handbook of theory, assessment, and treatment. Washington: American Psychological Association.

Clohessy, S., \& Ehlers, A. (1999). PTSD symptoms, response to intrusive memories and coping in ambulance service workers. The British Journal of Clinical Psychology, 38, 251-265.

Davey, G. C. L., Jubb, M., \& Cameron, C. (1996). Catastrophic worrying as a function of changes in problem-solving confidence. Cognitive Therapy and Research, 20, 333-344.

Davey, G. C. L., \& Levy, S. (1998). Catastrophic worrying: Personal inadequacy and a perseverative iterative style as features of the catastrophizing process. Journal of Abnormal Psychology, 107, 576586.

Davey, G. C. L., \& Levy, S. (1999). Internal statements associated with catastrophic worrying. Personality and Individual Differences, 26, 21-32.

Ehlers, A., \& Clark, D. M. (2000). A cognitive model of posttraumatic stress disorder. Behaviour Research and Therapy, 38, 319-345.

Ehlers, A., Hackmann, A., \& Michael, T. (2004). Intrusive re-experiencing in post-traumatic stress disorder: Phenomenology, theory, and therapy. Memory, 12, 403-415.

Ehlers, A., Mayou, R. A., \& Bryant, B. (1998). Psychological predictors of chronic posttraumatic stress disorder after motor vehicle accidents. Journal of Abnormal Psychology, 107, 508-519.

Ehlers, A., \& Steil, R. (1995). Maintenance of intrusive memories in posttraumatic stress disorder: A cognitive approach. Behavioural and Cognitive Psychotherapy, 23, 217-249.

Ehring, T., Ehlers, A., \& Glucksman, E. (in press). Contribution of cognitive factors to the prediction of posttraumatic stress disorder, phobia and depression after road traffic accidents. Behaviour Research and Therapy.

Ehring, T., Ehlers, A., \& Glucksman, E. (in preparation). A prospective longitudinal study of posttraumatic stress disorder, phobia, and depression after road traffic accidents.

Evans, C., Ehlers, A., Mezey, G., \& Clark, D. M. (in press). Intrusive memories and ruminations related to violent crime among young offenders: Phenomenological characteristics. Journal of Traumatic Stress.

First, M. B., Spitzer, R. L., Gibbon, M., \& Williams, J. B. W. (1996). Structured clinical interview for DSM-IV axis I disorders. Washington: American Psychiatric.

Foa, E. B., Cashman, L., Jaycox, L., \& Perry, K. (1997). The validation of a self-report measure of posttraumatic stress disorder: The Posttraumatic Diagnostic Scale. Psychological Assessment, 9, 445-451.

Foa, E. B., \& Kozak, M. J. (1986). Emotional processing of fear: Exposure to corrective information. Psychological Bulletin, 99, 20-35.

Fresco, D. M., Frankel, A. N., Mennin, D. S., Turk, C. L., \& Heimberg, R. G. (2002). Distinct and overlapping features of rumination and worry: The relationship of cognitive production to negative affective states. Cognitive Therapy and Research, 26, 179-188.

Greenberg, M. A. (1995). Cognitive processing of traumas: The role of intrusive thoughts and reappraisals. Journal of Applied Social Psychology, 25, 1262-1296.

Hackmann, A., Ehlers, A., Speckens, A., \& Clark, D. M. (2004). Characteristics and content of intrusive memories in PTSD and their changes with treatment. Journal of Traumatic Stress, 17, 231-240.

Halligan, S. L., Clark, D. M., \& Ehlers, A. (2002). Cognitive processing, memory, and the development of PTSD symptoms: Two experimental analogue studies. Journal of Behavior Therapy and Experimental Psychiatry, 33, 73-89.

Harvey, A. G., \& Greenall, E. (2003). Catastrophic worry in primary insomnia. Journal of Behavior Therapy and Experimental Psychiatry, 34, 11-23.

Harvey, A. G., Watkins, E., Mansell, W., \& Shafran, R. (2004). Cognitive behavioural processes across psychological disorders. Oxford, UK: Oxford University Press.

Holman, E. A., \& Silver, R. C. (1998). Getting "stuck" in the past: Temporal orientation and coping with trauma. Journal of Personality and Social Psychology, 74, 1146-1163.

Joseph, S., Williams, R., \& Yule, W. (1997). Understanding post-traumatic stress: A psychosocial perspective on PTSD and treatment. Chichester, UK: Wiley.

King, L. A., \& Pennebaker, J. W. (1996). Thinking about goals, glue, and the meaning of life. In R. S. Wyer (Ed.), Ruminative thoughts (pp. 97-106). Mahwah, NJ: Erlbaum.

Luminet, O. (2004). Measurement of depressive rumination and associated constructs. In C. Papageorgiou \& A. Wells (Eds.), Depressive rumination: Nature, theory and treatment (pp. 187-215). Chichester, UK: Wiley.

Lyubomirsky, S., \& Tkach, C. (2004). The consequences of dysphoric rumination. In C. Papageorgiou, \& A. Wells (Eds.), Depressive rumination: Nature, theory and treatment (pp. 21-41). Chichester, UK: Wiley. 
Martin, L. L., \& Tesser, A. (1996). Some ruminative thoughts. In R. S. Wyer (Ed.), Ruminative thoughts (pp. 1-47). Mahwah, NJ: Erlbaum.

Mayou, R. A., Bryant, B. M., \& Ehlers, A. (2001). Prediction of psychological outcomes one year after a motor vehicle accident. The American Journal of Psychiatry, 158, 1231-1238.

McNally, R. J., Bryant, R. A., \& Ehlers, A. (2003). Does early psychological intervention promote recovery from posttraumatic stress? Psychological Science in the Public Interest, 4, 45-79.

Michael, T., Ehlers, A., Halligan, S., \& Clark, D. M. (2005). Unwanted memories of assault: What intrusion characteristics predict PTSD? Behaviour Research and Therapy, 43, 613-628.

Michael, T., Halligan, S. L., Clark, D. M., \& Ehlers, A. (in press). Rumination in posttraumatic stress disorder. Depression and Anxiety.

Murray, J., Ehlers, A., \& Mayou, R. A. (2002). Dissociation and posttraumatic stress disorder: Two prospective studies of motor vehicle accident survivors. The British Journal of Psychiatry, 180, 363368.

Nolen-Hoeksema, S. (2004). The response styles theory. In C. Papageorgiou \& A. Wells (Eds.), Depressive rumination: Nature, theory and treatment (pp. 107-123). Chichester, UK: Wiley.

Nolen-Hoeksema, S., \& Morrow, J. (1991). A prospective study of depression and posttraumatic stress symptoms after a natural disaster: The 1989 Loma Prieta earthquake. Journal of Personality and Social Psychology, 61, 115-121.

O’Donnell, M. L., Creamer, M., Pattison, P., \& Atkin, C. (2004). Psychiatric morbidity following injury. The American Journal of Psychiatry, 161, 507-514.

Paivio, A. (1986). Mental representation: A dual coding approach. New York: Oxford University Press.

Papageorgiou, C., \& Wells, A. (2003). An empirical test of a clinical metacognitive model of rumination and depression. Cognitive Therapy and Research, 27, 261-273.

Papageorgiou, C., \& Wells, A. (2004). Depressive rumination: Nature, theory and treatment. Chichester: Wiley.

Provencher, M. D., Freeston, M. H., Dugas, M. J., \& Ladouceur, R. (2000). Catastrophizing assessment of worry and threat schemata among worriers. Behavioural and Cognitive Psychotherapy, 28, 211224.

Schnurr, P. P., Lunney, C. A., \& Sengupta, A. (2004). Risk factors for the development versus maintenance of posttraumatic stress disorder. Journal of Traumatic Stress, 17, 85-95.

Segerstrom, S. C., Tsao, J. C. I., Alden, L. E., \& Craske, M. G. (2000). Worry and rumination: Repetitive thought as a concomitant and predictor of negative mood. Cognitive Therapy and Research, 24, 671688.

Speckens, A. E. M., Ehlers, A., Hackmann, A., Ruths, F., \& Clark, D. M. (submitted). Intrusive memories and rumination in patients with posttraumatic stress disorder: A phenomenological comparison.

Startup, H. M., \& Davey, G. C. L. (2001). Mood as input and catastrophic worrying. Journal of Abnormal Psychology, 110, 83-96.

Startup, H. M., \& Davey, G. C. L. (2003). Inflated responsibility and the use of stop rules for catastrophic worrying. Behaviour Research and Therapy, 41, 495-503.

Steil, R., \& Ehlers, A. (2000). Dysfunctional meaning of posttraumatic intrusions in chronic PTSD. Behaviour Research and Therapy, 38, 537-558.

Stöber, J. (1998). Worry, problem elaboration and suppression of imagery: The role of concreteness. Behaviour Research and Therapy, 36, 751-756.

Stöber, J., \& Borkovec, T. D. (2002). Reduced concreteness of worry in generalized anxiety disorder: Findings from a therapy study. Cognitive Therapy and Research, 26, 89-96.

Stöber, J., Tepperwien, S., \& Staak, M. (2002). Worrying leads to reduced concreteness of problem elaborations: Evidence for the avoidance theory of worry. Anxiety, Stress, and Coping, 13, 217-227.

Teasdale, J. D. (1999). Emotional processing, three modes of mind and the prevention of relapse in depression. Behaviour Research and Therapy, 37, S53-S77.

Vasey, M. W., \& Borkovec, T. D. (1992). A catastrophizing assessment of worrisome thoughts. Cognitive Therapy and Research, 16, 505-520.

Watkins, E. (2004). Adaptive and maladaptive ruminative self-focus during emotional processing. Behaviour Research and Therapy, 42, 1037-1052.

Watkins, E., \& Mason, A. (2002). Mood as input and rumination. Personality and Individual Differences, 32, 577-587.

Wells, A. (1995). Meta-cognition and worry: A cognitive model of generalized anxiety disorder. Behavioural and Cognitive Psychotherapy, 23, 301-320. 